\title{
Microparticles in systemic sclerosis: potential new biomarker?
}

Sandra Maximiano de Oliveira (UNIFESP, São Paulo, SP, Brasil), Ighor Luiz Azevedo Teixeira (UNIFESP, São Paulo, SP, Brasil), Carolina Nunes França (UNISA/UNIFESP, São Paulo, SP, Brasil), Cristiane Kayser (UNIFESP, São Paulo, SP, Brasil), Lucas Garcia Biagi (UNIFESP, São Paulo, SP, Brasil), Maria Cristina de Oliveira Izar (UNIFESP, São Paulo, SP, Brasil)

\section{BACKGROUND}

Systemic sclerosis (SSc) is a rare autoimmune disease, with high morbidity and mortality, characterized by vascular damage, immune dysregulation and progressive tissue fibrosis. Microparticles (MP) are membrane-derived vesicles released from cells undergoing activation or apoptosis. MP work as immunologically active molecules regulating inflammatory and immune responses, coagulation, homeostasis and intercellular communication. The objective of this work is to compare serum levels of MP derived from platelets (PMP), endothelial cells (EMP) and monocytes (MMP) between scleroderma patients and healthy controls.

\section{MATERIALS AND METHODS}

In this cross-sectional study, 23 SSc patients and 14 age- and sex-matched healthy controls were recruited. Plasma MP were quantified by flow cytometry after staining with fluorescent cell-specific monoclonal antibodies: CD42+/31+ for PMP, CD105+ for EMP, and CD14+ for MMP.

\section{RESULTS}

All patients fulfilled the 2013 ACR/EULAR classification criteria for SSc. Demographic characteristics of studied population are summarized on table 1. All types of MP were increased in SSc patients compared with healthy controls (mean \pm SD): $80.12 \% \pm 3.21 \%$ versus $68.0 \% \pm 6.32 \%$ for PMP, $p=0.10 ; 48.62 \% \pm 1.33 \%$ versus $26.82 \% \pm 4.0 \%$ for EMP, $p<0.0001$; and $4.21 \% \pm 0.29 \%$ versus $0.83 \% \pm 0.27 \%$ for $M M P, p<0.0001$ ), with significant increase in MP derived from endothelial cells and monocytes (figure 1).

\section{CONCLUSION}

Serum level of MP derived from platelets, endothelial cells and monocytes are elevated in SSc patients, indicating that these cells are activated in SSc and that MP may play a role in scleroderma pathogenesis. This study was supported by FAPE-SBR. 